\title{
Strong light-matter coupling in dielectric metasurfaces
}

\author{
Gabriel W. Castellanos ${ }^{1, *}$, Shunsuke Murai ${ }^{2}$, T.V. Raziman ${ }^{1}$, Shaojun Wang ${ }^{1,3}$, Mohammad Ramezani ${ }^{1}$, Alberto G. Curto ${ }^{1}$, \\ and Jaime Gómez Rivas ${ }^{1}$ \\ ${ }^{1}$ Department of Applied Physics and Institute for Photonic Integration, Eindhoven University of Technology, P.O. Box 513, 5600 MB \\ Eindhoven, The Netherlands \\ ${ }^{2}$ Department of Material Chemistry, Graduate School of Engineering, Kyoto University, Nishikyo-ku, Kyoto 615-8510, Japan \\ ${ }^{3}$ MOE Key Laboratory of Modern Optical Technologies and Jiangsu Key Laboratory of Advanced Optical Manufacturing Technologies, \\ School of Optoelectronic Science and Engineering, Soochow University, Suzhou 215006, China
}

\begin{abstract}
We demonstrate the strong coupling between excitons in organic molecules and all-dielectric metasurfaces formed by arrays of silicon nanoparticles supporting Mie surface lattice resonances (MSLRs). Compared to Mie resonances in individual nanoparticles, MSLRs have extended mode volumes and much larger quality factors, which enables to achieve collective strong coupling with very large coupling strengths and Rabi energies. Moreover, due to the electric and magnetic character of the MSLR given by the Mie resonance, we show that the hybridization of the exciton with the MSLR results in exciton-polaritons that inherit this character as well. Our results demonstrate the potential of all-dielectric metasurfaces as novel platform to investigate and manipulate exciton-polaritons in low-loss polaritonic devices.
\end{abstract}

\section{Introduction}

The use of dielectric metasurfaces supporting Mie resonances as optical cavities to control light-matter interactions has triggered many interesting works in the last years. Due to the low absorption losses and the presence of resonances with both electric and magnetic character, dielectric metasurfaces have an exciting prospect for the development of novel applications.

However, such work has been limited to the control of emission, a weak light-matter coupling phenomenon. Achieving the strong coupling regime with Mie resonances has remained challenging due to their moderate field enhancements and large radiative losses.[1, 2] Strong coupling results in the hybridization of light and matter into quasiparticles known as excitonpolaritons, with remarkable properties such as enhanced transport, long-range energy transfer, condensation, and non-linear response.

Here, we demonstrate experimentally the strong coupling between excitons in organic molecules and alldielectric metasurfaces formed by arrays of polycrystalline silicon nanoparticles supporting Mie surface lattice resonances (MSLRs) with electric and magnetic character.[3] We first discuss the bare MSLRs in the array without molecules and explain the origin of their electric and magnetic character. Next, we deposit an organic dye on the array and show the formation of polaritonic states. Using a coupled oscillator model, we fit these states and extract the values of the Rabi energy. Finally, using simulations we discuss the character of the excitonpolaritons formed in this system.

\section{Mie surface lattice resonances (MSLRs)}

MSLRs emerge from the enhanced radiative coupling between the Mie resonances of nanoparticles through inplane diffracted orders known as Rayleigh anomalies (RAs). Because of the low-loss characteristic of diffracted orders, MSLRs have large quality factors. Furthermore, due to the collective nature of the resonance, MSLRs have extended mode volumes that allow interaction with many emitters.

Our array has a periodicity $\mathrm{a}=400 \mathrm{~nm}$ and the particles have a diameter $\mathrm{d}=120 \mathrm{~nm}$ with height $\mathrm{h}=90 \mathrm{~nm}$. To characterize the optical response of the MSLRs we measure the extinction as function of the incident in-plane moment $\mathbf{k}_{\|}\left(\mathrm{k}^{2}=\mathrm{k}_{\|}{ }^{2}+\mathrm{k}_{\mathrm{z}}{ }^{2}\right)$ of the metasurface with a deposited layer of poly(methyl methacrylate) (PMMA) with a thickness of $t=290 \pm 10 \mathrm{~nm}$ (see Figure 1a and 1b). The extinction is defined as $1-\mathrm{T}_{0}$, where $\mathrm{T}_{0}$ is the zerothorder transmission. The measurements for TE (Figure 1a) and TM (Figure 1b) polarizations reveal several dispersive modes with Fano lineshapes, which correspond to the bare MSLRs arising from the RAs (indicated by the grey lines).

MSLRs can have an electric or magnetic character that is given by the presence of both electric and magnetic dipole resonances in the individual silicon nanoparticles. The character of the MSLR can be understood with the help of the bottom diagram that shows the preferential coupling between the excited dipoles for a given polarization of the incident light field.

\footnotetext{
* Corresponding author: g.w.castellanos.gonzalez@tue.nl
} 


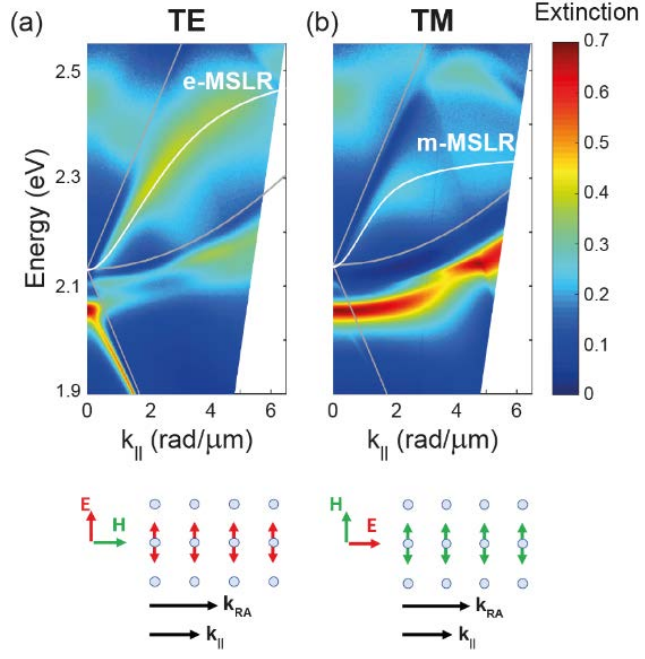

Fig. 1. Extinction of the MSLRs as function of the incident inplane moment $\mathbf{k}_{\|}$for TE (a) and TM (b) polarizations. The RAs are identified by the grey lines. The fit to the MSLRs of interest corresponds with the white solid curves. This corresponds with an electric MSLR (e-MSLR) and a magnetic MSLR (m-MSLR) for TE and TM polarizations, respectively. The character of the MSLR can be understood with the help of the bottom diagram that shows the preferential coupling between the excited dipoles for a given polarization of the incident light field.

\section{Strong light-matter coupling with MSLRs}

The interaction between the dye molecules and the MSLR corresponds to the collective coupling between multiple emitters with a common cavity mode. This collective interaction results in a coupling strength proportional to the number of emitters within the mode volume of the cavity.

Consequently, to achieve strong coupling we deposit a layer of PMMA doped with organic molecules in a concentration of $35 \mathrm{wt} \%$. This molecule is characterized by a main electronic transition, that we label as $\mathrm{X}_{1}$, and a vibronic replica of the main electronic transition, that we label as $\mathrm{X}_{2}$. Due to the different dispersion of the MSLRs, only the fitted MSRLs in Figure 1a and 1b interact with the molecule. This corresponds with an electric MSLR (eMSLR) and a magnetic MSLR (m-MSLR) for the TE and TM polarizations, respectively. The extinction as function of the incident in-plane moment $\mathbf{k}_{\|}$in Figure 2 shows the formation of exciton-polariton states which have a different dispersion compared with that of the bare cavity and bare excitons. We analyse the new eigenstates using a coupled oscillator model that defines the interaction between the MSLRs (the cavity mode) and the electronic transitions as function of the incident in-plane momentum. The fitted lower, middle and upper polariton states (LP, MP and UP, respectively) are identified in Figure $2 \mathrm{a}$ and $2 \mathrm{~b}$ by the white dashed lines. Extracting the Rabi energy from the fitting we obtain the value of $\hbar \Omega \sim 200 \mathrm{meV}$ for the splitting between the LP and MP for $\mathrm{TE}$, and $\hbar \Omega \sim 200 \mathrm{meV}$ for the splitting between the LP and UP in TM. To claim strong coupling from an experimental optical spectrum, as is the case with the extinction, it is common to use the condition of clear visibility of the exciton-polariton states in the spectrum, which requires that $(2 g)^{2}>\left(\gamma_{M S L R}^{2}+\gamma_{\text {exciton }}^{2}\right) / 2$. Comparing the obtained values of the Rabi energy with the losses of the system, we conclude that the strong coupling has been achieved in both TE and TM polarizations.

Finally, the electric or magnetic character of the MSLR not only influences the interaction strength with the dye, but it also determines the character of the hybridization. Our simulations (Figures $2 \mathrm{c}$ and $2 \mathrm{~d}$ ) of the spatial distribution of the electric field show that due to the extended mode volume of the MSLR, this electric and magnetic character is inherited as well in the excitonpolariton formed at the strong coupling regime.
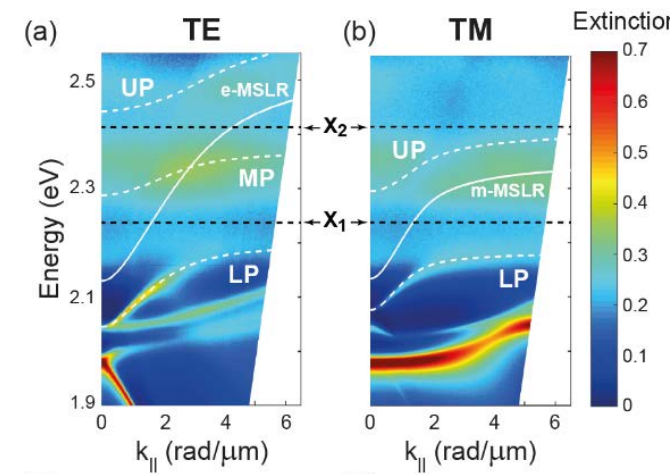

(c)
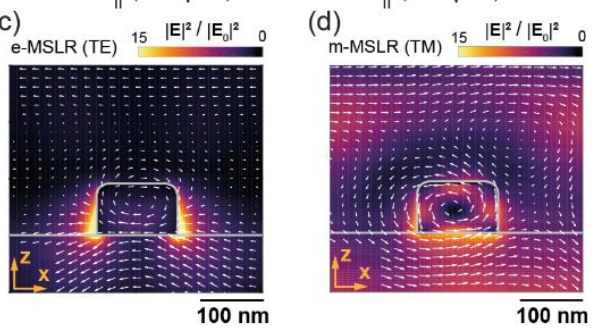

Fig. 2. Extinction in presence of organic dye as function of the incident in-plane moment $\mathbf{k}_{\|}$for TE (a) and TM (b) polarizations. The formation of lower, middle and upper polaritons (LP, MP and UP respectively) and their fit (white dashed lines) indicates the strong coupling between the excitons and the MSRLs. In (c) and (d) the spatial distribution of the electric fields shows the electric and magnetic character of the MSLR is shown for each polarization. The color scale represents the intensity of the electric field $\left(|\mathbf{E}|^{2} /\left|\mathbf{E}_{0}\right|^{2}\right)$ and the white arrows represent the vectorial projection of the electric field in the xz-plane.

These results demonstrate the potential of alldielectric metasurfaces as a novel platform to investigate and manipulate exciton-polaritons in low-loss polaritonic devices.

\section{References}

1. F. Todisco, R. Malureanu, C. Wolff, P. Gonçalves, A. Roberts, N. Mortensen, C. Tserkezis, Nanophotonics 9 (4), 803-814 (2020).

2. R. Heilmann, AI. Väkeväinen, JP. Martikainen, P. Törmä, Nanophotonics 9 (2), 267-276 (2020).

3. G.W. Castellanos, S. Murai, T.V. Raziman, S. Wang, M. Ramezani, A.G. Curto, and J. Gómez Rivas, ACS Photonics (accepted). 